\title{
Crystallization Instability in Glass-Forming Mixtures
}

\author{
Trond S. Ingebrigtsen, ${ }^{1,2}$ Jeppe C. Dyre, ${ }^{2}$ Thomas B. Schrøder, ${ }^{2}$ and C. Patrick Royall ${ }^{3}$ \\ ${ }^{1}$ Department of Fundamental Engineering, Institute of Industrial Science, University of Tokyo, \\ 4-6-1 Komaba, Meguro-ku, Tokyo 153-8505, Japan \\ ${ }^{2}$ Glass and Time, IMFUFA, Department of Science and Environment, Roskilde University, \\ P.O. Box 260, DK-4000 Roskilde, Denmark \\ ${ }^{3}$ H.H. Wills Physics Laboratory, Tyndall Avenue, Bristol, BS8 1TL, United Kingdom, School of Chemistry, \\ University of Bristol, Cantock's Close, Bristol, BS8 ITS, United Kingdom, \\ and Centre for Nanoscience and Quantum Information, \\ Tyndall Avenue, Bristol, BS8 1FD, United Kingdom
}

(Received 18 February 2019; revised manuscript received 16 May 2019; published 1 August 2019)

\begin{abstract}
Understanding the mechanisms by which crystal nuclei form is crucial for many phenomena such as gaining control over crystallization in glass-forming materials or accurately modeling rheological behavior of magma flows. The microscopic nature of such nuclei, however, makes their understanding extremely hard in experiments, while computer simulations have hitherto been hampered by short timescales and small system sizes. Here we use highly efficient graphics processing unit simulation techniques to address these challenges. The larger systems we access reveal a general nucleation mechanism in mixtures. In particular, we find that the supercooled liquid of a prized atomistic model glass former (Kob-Andersen model) is inherently unstable to crystallization, i.e., that nucleation is unavoidable on the structural relaxation timescale, for system sizes of 10000 particles and larger. This is due to compositional fluctuations leading to regions composed of one species that are larger than the critical nucleus of that species, which rapidly crystallize. We argue that this mechanism provides a minimum rate of nucleation in mixtures in general, and show that the same mechanism pertains to the metallic glass former copper zirconium $(\mathrm{CuZr})$.
\end{abstract}

DOI: 10.1103/PhysRevX.9.031016

\section{INTRODUCTION}

Crystallization in supercooled liquids has profound implications in fields as diverse as the development of amorphous materials [1], magma flows in volcanos [2], and aqueous solutions of ions [3]. Materials in question include metallic, inorganic, and chalcogenide glass formers, where mixtures of a number of different constituents have the effect of suppressing or controlling crystallization [4]. Alas, this tendency to crystallize places stringent limits on the size of the pieces of amorphous material that can be formed: large pieces are more likely to undergo crystal nucleation [5,6]. This "Achilles heel" of glass formation thus limits the exploitation of metallic glasses, for example, whose superior mechanical properties otherwise hold great promise [7].

It is clear that any liquid cooled below its freezing point must, for a sufficiently large system, nucleate [8,9].

Published by the American Physical Society under the terms of the Creative Commons Attribution 4.0 International license. Further distribution of this work must maintain attribution to the author(s) and the published article's title, journal citation, and DOI.
Subject Areas: Chemical Physics, Materials Science, Soft Matter
However, the practical limits of cooling rate versus system size required for vitrification are not known in general. In additional to these practical considerations, crystallization is one solution to the Kauzmann paradox of vanishing configurational entropy upon which a number of theories of the glass transition rest $[4,10]$ : crystallization avoids the need to invoke any particular theoretical description of divergent viscosity in amorphous materials [11-13].

It is known empirically that increasing the number of constituent species and introducing a size disparity among these components, together with a negative heat of mixing, tends to suppress nucleation - this has been the guiding principle in the development of bulk metallic glasses [5]. However, despite recent innovative approaches using model systems [14,15] and novel sampling techniques $[16,17]$, there is still a lack of fundamental understanding of the mechanisms by which glass-forming mixtures crystallize.

Here we consider a crystallization mechanism that is always present when a glass former is produced by mixing constituents which by themselves are poor glass formers, as is often the case. We therefore expect this mechanism to be remarkably widespread. In particular, compositional fluctuations in the supercooled liquid lead to regions containing 
just a single constituent, and eventually such a region will occur that is large enough — and long-lived enough — that it will nucleate a crystal of that one species. Of course, depending on the specific mixture, there may be other, faster, nucleation mechanisms. Nucleation by compositional fluctuations nevertheless provides a lower bound for the nucleation rate in mixtures. We emphasize that compositional fluctuations occur even in the absence of any underlying demixing behavior driven by a thermodynamic transition. In fact, the first mixture we investigate is specifically designed not to demix, using a nonadditive attractive cross interaction between the two species. Thus the compositional fluctuations we consider are distinct from enhanced crystal nucleation rates due, for example, to density fluctuations related to a nearby critical point $[18,19]$. Clearly, our analysis falls within the concept of the Ostwald rule of stages, suitably generalized to mixtures [20]. In this context, we emphasize that the crystals formed through such compositional fluctuations will in general not be thermodynamically stable.

Having argued for compositional fluctuations as a relevant mechanism for crystal nucleation, we turn our attention to situations in which this mechanism may dominate. We begin with the Kob-Andersen (KA) binary Lennard-Jones mixture. Since its inception in 1994, this model, based on the metallic glass former nickel phosphorous, has been a mainstay of model systems with which to tackle the glass transition [21]. Prized for its simplicity, speed of computation, and its stability against crystallization, the KA model is among the most widely used atomistic glass formers in computer simulations. It is only recently, with the advance of high-performance graphics processing unit (GPU) computing, that the KA model has been crystallized by direct simulation [15], where an estimate was made of the nucleation rate at a single temperature and system size. Here, instead, we carry out large-scale simulations and focus on the mechanism for crystallization.

Our results reveal that nucleation in the KA model is induced by composition fluctuations as discussed above. The KA model is thus representative of systems crystallizing via this mechanism, and we expect our results to have profound consequences for the glass-forming ability of mixtures, such as metallic glasses [7] and oxides [22]. We illustrate the generality of our results by presenting results for the model metallic glass former $\mathrm{Cu}_{x} \mathrm{Zr}_{1-x}$ using a range of compositions from $x=0.15$ to 0.645 .

\section{FREEZING IN THE KOB-ANDERSEN MODEL GLASSFORMER}

We begin the presentation of our results by studying crystallization in the KA mixture using a global structural analysis for $\rho=1.204$ and $T=0.40$ in Figs. 1(b) and 1(c). We use the NVT ensemble with a Nose-Hoover thermostat [23]. Figures 1(b) and 1(c) show, respectively, the time evolution of the population of liquid local structures (bicapped square antiprisms) and crystalline structures for system sizes of $N=10000$ and $N=100000$. Here and henceforth we scale time by the structural relaxation time $\tau_{\alpha}$. For $T=0.40$, we have that the structural relaxation time $\tau_{\alpha}=2.91 \times 10^{5}$ simulation time units. A snapshot of a crystal nucleus, composed predominantly of the majority $A$ species, is shown in Fig. 1(a). We identify particles in liquid locally favored structures (LFS) and fcc, hcp crystalline regions with the topological cluster classification (TCC) algorithm [24] and bcc crystalline regions with bond-orientational order (BOO) parameters $[25,26]$. Our choice of order parameter is motivated by the ability of the TCC to identify the liquid local structure (and hcp and fcc), and we have in any case confirmed that our results for identification of the crystal structures are (a)

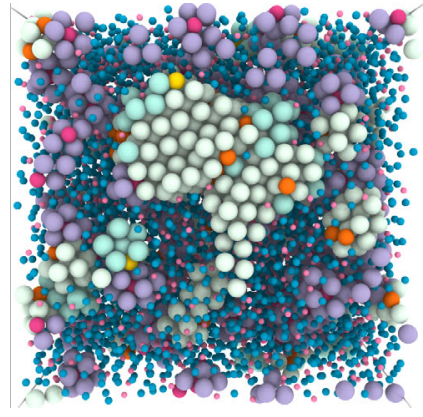

(b)

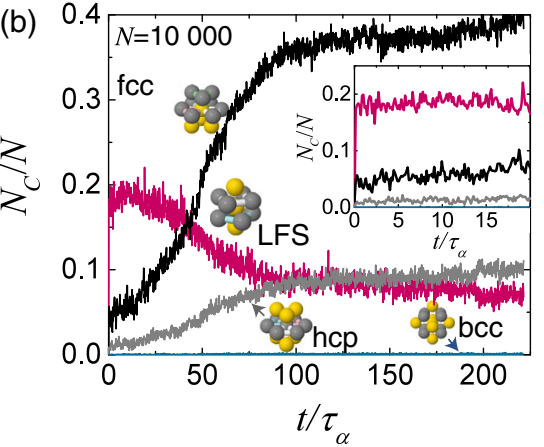

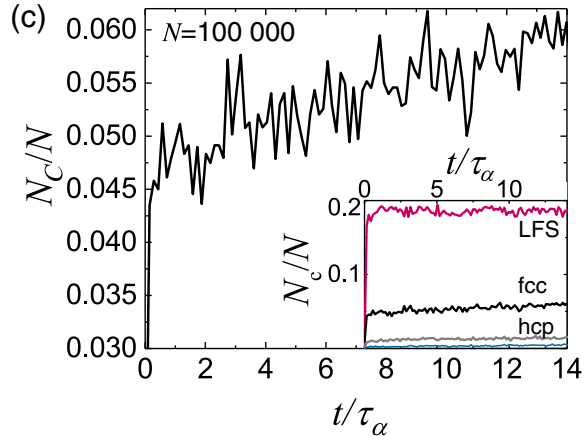

FIG. 1. Structural analysis of crystallization in the Kob-Andersen glass former. (a) Particle snapshot at time $t \approx 36 \tau_{\alpha}$ at $T=0.40$ and $N=10000$. We observe a fcc-dominated crystallite of $A$ particles. Light green and yellow particles are fcc for $A$ and $B$ particles, respectively, light blue and orange are hcp, purple and dark pink are bicapped antiprism liquid locally favored structure (LFS), and dark blue and light pink particles are liquid. (b) Population of local structures as a function of time reveals rapid crystallization of fcc and hcp for $N=10000$. bcc is found in very small quantities, and the bicapped square antiprism liquid LFS (which is incompatible with fcc and hcp) is also shown. The inset shows that at short times $\left(\lesssim 5 \tau_{\alpha}\right)$, we cannot infer any crystal growth within the fluctuations. (c) Even more rapid crystallization occurs when $N=100000$; here irreversible growth in the fcc population is found within one relaxation time. Inset: Liquid LFS and hcp populations are compared with fcc population, as shown in the main figure. 
very similar between the two methods (see the Appendix for simulation details and order parameters).

We see from Fig. 1(b) that the liquid begins to freeze on a timescale of a few structural relaxation times $\tau_{\alpha}$. Thus, for these parameters of $T=0.40$ and $N=10000$, it is hard to regard the KA mixture as anything but a remarkably poor glass former. We further see that the population of the LFS in the liquid, the bicapped square antiprism [shown in Fig. 1(b)], reduces upon crystal growth in much the same way as in one-component hard spheres where the liquid LFS competes with the crystal symmetry [27].

Here, of course, we have a binary system, but the predominant crystal structures we find are fcc and hcp of the large $A$ species only, and very little mixed $A B$ bcc. The lack of bcc is consistent with predictions that the crystal nucleation barrier is much higher relative to fcc [28] and with the equilibrium KA phase diagram [29]. For the KA model, we therefore neglect the bcc structure and focus on the hcp and fcc crystals in the following.

In Fig. 1(b), we see that there seems to be very little incubation time. However, close inspection [Fig. 1(b), inset] reveals that for timescales of a few $\tau_{\alpha}$, the fluctuations in crystal population are larger than the increase, so the liquid may in fact be regarded as metastable on short timescales. In Fig. 1(c), we show that upon a further increase of system size, to $N=100000$, this short time metastability vanishes, and the crystal nuclei grow immediately.

We now consider the formation of critical crystal nuclei and estimate their size. In Figs. 2(a) and 2(b), we show the number of particles $N_{\text {xtal }}$ in the largest connected region of crystal particles (hcp or fcc) for different system sizes. Here we select a run with a relatively long incubation period [Fig. 2(a)]. We see that the crystal regions are smaller than 100 particles for around $40 \tau_{\alpha}$ before growing. These data enable us to infer a critical nucleus size of approximately 50-100 particles for $T=0.40$. Figure 2(b) shows the run at $N=100000$, where crystal growth is immediate and thus it is difficult to infer a critical nucleus size in this case.
Next, we consider the statistics of nucleation in the KA glass former. From the ten runs we performed for $N=10000$ and $T=0.40$ (all of which crystallized), we determine the mean nucleation time from $\tau_{X}=$ $\sum_{i=1}^{n} t_{X(i)} / n$, where $n$ is the total number of simulations, to be $\tau_{X}=38.4 \pm 26.8 \tau_{\alpha}$ (the error is the standard deviation). Here $t_{X(i)}$ is the time when the size of the largest crystal region reaches, and does not subsequently drop below, 100 particles. At higher temperatures, the driving force for crystallization is of course reduced, but the dynamics is much faster. We find that the system does crystallize at higher temperatures (we probed up to $T=0.45$ ) but that not all the runs do so. In this case, we determine the mean nucleation time for each state point following the method of Ref. [30]. In particular, we presume that nucleation is exponentially distributed in time, such that the probability of a nucleation event happening at time $t$ is $p(t)=1 / \tau_{X} \exp \left(-t / \tau_{X}\right)$. The probability that a given run of length $t_{\text {run }}$ crystallizes is then $\int_{0}^{t_{\text {run }}} p(t) d t=1-\exp \left(-t_{\text {run }} / \tau_{X}\right)$. The fraction of runs that crystallized then gives us $\tau_{X}$. Errors are estimated by considering the case that one more, or one fewer, simulation runs underwent crystallization. While more sophisticated analyses have been developed, which enable accurate determination of the critical nucleus size [31], even with the considerable computational resources we have used, it has only been possible to carry out ten runs per state point. This limits the extent to which we can implement such methods.

We see from Fig. 3(a) that, when scaled by the relaxation time, the time to nucleate drops rapidly with temperature at $N=10000$, and that well before the dynamical divergence temperature predicted from a Vogel-Fulcher-Tamman fit to the temperature dependence of the relaxation time ( $T_{0} \approx 0.30$, see Supplemental Material [32]), the nucleation time $\tau_{X}$ is expected to fall below $\tau_{\alpha}$ at $T \approx 0.38$. Moreover in the range $T \lesssim 0.43$, we find an exponential scaling with temperature, $\tau_{X} / \tau_{\alpha} \sim e^{A T}$ with $A \approx 97$. Of course, this
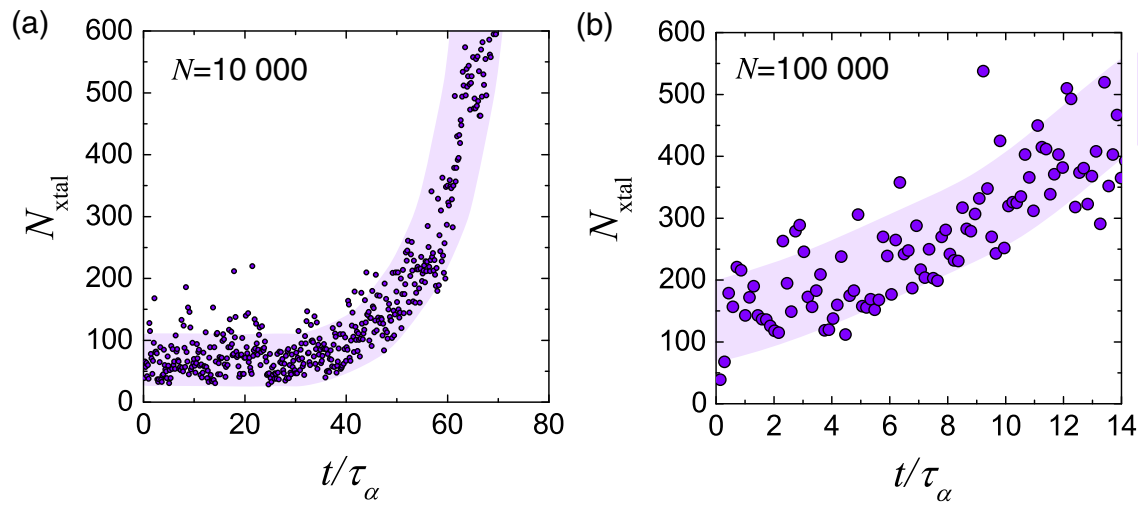

FIG. 2. Time evolution of the largest crystalline region in the KA mixture. All data are for temperature $T=0.40$ and shading is to guide the eye. (a) Run with incubation period of around $40 \tau_{\alpha}$ prior to growth of crystalline region $(N=10000)$. (b) Immediate crystal growth at $N=100000$. 

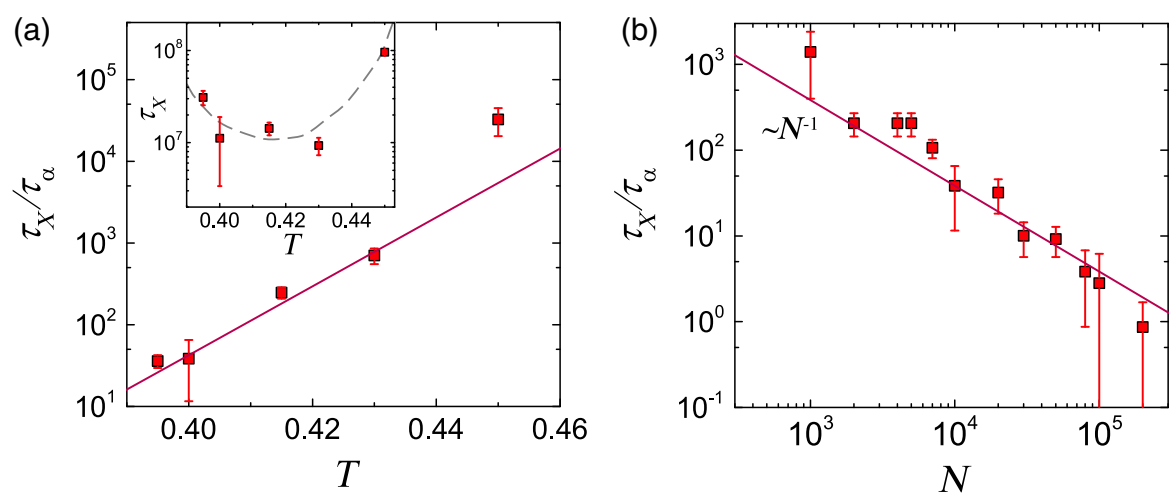

FIG. 3. Nucleation times $\tau_{X}$ with respect to temperature and system size in the KA mixture. (a) Nucleation time scaled by the relaxation time $\tau_{\alpha}$ as a function of temperature $T$ at $N=10000$. Line is a fit to $\tau_{X} / \tau_{\alpha} \sim e^{A T}$, with $A \approx 97$. Inset: Nonscaled nucleation times $\tau_{X}$ with a curve to guide the eye (dashed line). (b) Nucleation times as a function of system size at $T=0.40$. Line represents expected $1 / N$ scaling for $\tau_{X}$ in the case of a constant nucleation rate. We determine $\tau_{X}$ as described in the text.

observation rests on only the four data points which we fit, but given the significant magnitude of the fall in $\tau_{X} / \tau_{\alpha}$ with temperature, we are confident that, were this trend to continue, the observation that for some $T>T_{0}, \tau_{X}<\tau_{\alpha}$ would hold.

When we simply plot the nucleation time in simulation time units [Fig. 3(a), inset], we make two observations. Firstly, the absolute nucleation time does not change hugely (around 1 order of magnitude) throughout the temperature range in question, while the relaxation time changes by 3 orders of magnitude. Secondly, there is an upturn at the lowest temperature that we consider, $T=0.395$. The reason for the minimum in $\tau_{X}(T)$ presented in the inset of Fig. 3(a) is then competition between the decrease in the average nucleation barrier (for a given system size) and the increase in relaxation time upon cooling, though we emphasize that this is only one data point and more statistics would be helpful to confirm this observation. In any case, this is dwarfed by the increase in relaxation time, so the scaled quantity $\tau_{X} / \tau_{\alpha}$ continues to drop. Turning to the system size dependence of nucleation in Fig. 3(b), we find, as expected, a system size scaling consistent with $\tau_{X} \sim 1 / N$. Note that in Fig. 3(b) we consider a single temperature, $T=0.40$, so that $\tau_{\alpha}$ does not enter into the scaling.

\section{COMPOSITION FLUCTUATIONS}

Next, we proceed to investigate the role of compositional fluctuations in crystallization. To quantify these, we use the order parameter illustrated in Fig. 4(b). We seek to find the largest region of liquid $A$ particles which is devoid of any $B$ particles. We presume that such a large compositional fluctuation would be most likely to drive crystallization.

Therefore, we use the following procedure for a given snapshot.

(1) We find the $A$ particle which is furthest away from the nearest $B$ particle.
(2) We define a sphere, centered on the $A$ particle, whose radius is its distance to the nearest $B$ particle.

(3) The number of particles in the sphere $n_{s}$ is taken as the current largest compositional fluctuation.

(4) We iterate to smaller $A B$ separations and hence smaller spheres, avoiding particles already contained in a previous sphere, and updating $n_{s}$ if a larger region is encountered.

Since there will be small fluctuations of crystal particles in the liquid, and we are looking for the largest region of liquid $A$ particles, we seek to avoid the effects of such $A$ particles in crystalline environments. Therefore, we accept a maximum of $10 \%$ of the particles in the sphere to be in a crystalline environment. The time evolution of the largest compositional fluctuation in each snapshot $n_{s}$ is shown in Fig. 4(c). We only sample where the system has yet to crystallize, under our criterion of a nucleus size of less than 100 particles. Simply because the system has not yet crystallized does not mean that its properties are stationary, as shown in Figs. 1 and 2. However, it is still instructive to apply the same metric for the larger systems as for the smaller systems (whose properties are stationary for timescales beyond the structural relaxation time), and this we do, with the caveat that the distributions are sampled from a nonstationary system.

In Fig. 4(a), we see that for the KA system at $T=$ 0.40 and $N=5000,10000$, and 100000 , the distribution of largest composition fluctuations $n_{s}$ of liquid $A$ particles has a significant dependence on the system size $N$. Two effects are apparent. Firstly, the typical size of compositional fluctuations increases with $N$. Secondly, the distribution has a "fat tail" indicating more fluctuations of larger $n_{s}$ than a symmetric distribution such as a Gaussian would predict. We note 50-100 particles was a rough estimate of the critical nucleus size and that fluctuations comparable to this are seen in the tails of the distributions. 

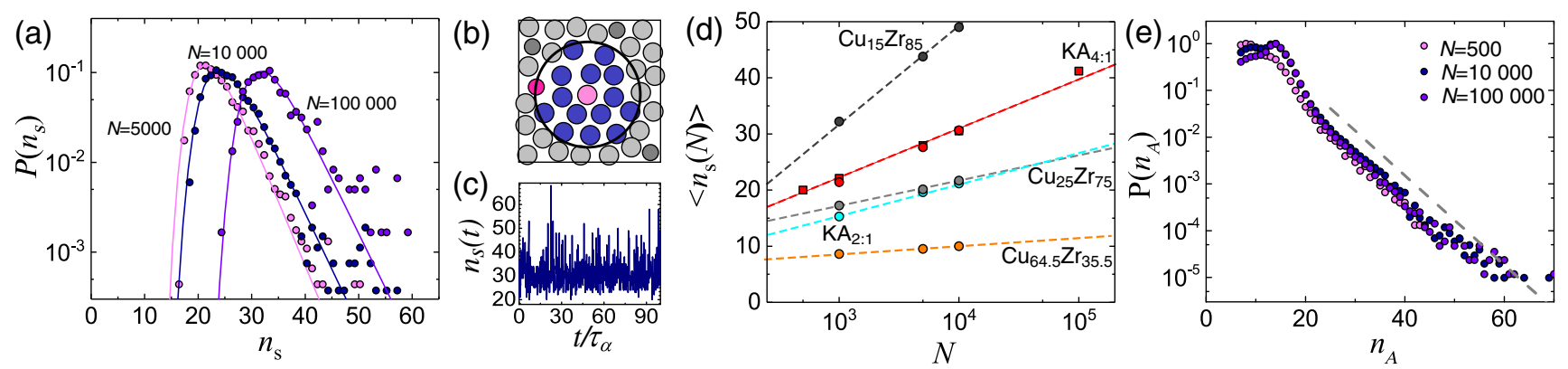

FIG. 4. Compositional fluctuations in $\mathrm{KA}$ and $\mathrm{CuZr}$ at various compositions. (a) Distributions of largest compositional fluctuations of $A$ particles $n_{s}$ for several system sizes $N$ in KA for $T=0.4$. Each system size is fitted with a Gumbel distribution (see text). (b) Schematic indicating the order parameter $n_{s}$ for compositional fluctuations. Central pink particle is the $A$ particle under consideration and dark pink particle the nearest $B$ particle. Blue particles are $A$ particles lying within the sphere as shown. The compositional fluctuation shown has $15 \mathrm{~A}$ particles. (c) Time evolution of largest compositional fluctuations $n_{s}$ in the liquid for the KA model with $N=10000$ and $T=0.4$. (d) Scaling of the median $\left\langle n_{s}\right\rangle$ calculated from fitted Gumbel distributions with system size; lines are fits (see text). Shown are data for the KA mixture at various compositions along with $\mathrm{Cu}_{x} \mathrm{Zr}_{1-x}$ metallic glass formers (see the Appendix for details). Circles are constant pressure data $(P=0)$ and squares are constant density data $(\rho=1.204)$. For the KA mixtures, constant pressure data are taken in the $N V T$ ensemble fixing the mean pressure at $\langle P\rangle=0$ and $T=0.80$ and constant density data are taken at $T=0.40 . \mathrm{Cu}_{x} \mathrm{Zr}_{1-x}$ is simulated in the NPT ensemble at $T=1270 \mathrm{~K}$ or $T=1500 \mathrm{~K}$ and $P=0$. (e) Distribution for all compositional fluctuations for several system sizes (4:1 KA mixture) at $T=0.40$. The distribution is independent of system size above $n_{A}>25$. Dashed line denotes exponential decay with decay constant $\lambda=0.22$ (see text).

\section{STATISTICS OF COMPOSITIONAL FLUCTUATIONS}

What can we say about the origin of the distribution of the largest compositional fluctuations in Fig. 4(a)? Let us suppose that the distribution of all fluctuations of $A$ particles is exponential, $P\left(n_{A}\right) \propto \exp \left(-n_{A} \lambda\right)$, where $n_{A}$ is the number of $A$ particles around a given $A$ particle that are closer than the nearest $B$ particle calculated for every $A$ particle. Here, $\lambda$ is the decay constant. The extreme values of such a distribution, i.e., those fluctuations large enough to initiate nucleation, should then follow a Gumbel distribution given by $P\left[z\left(n_{s}\right)\right] \propto e^{-\left(z+e^{-z}\right)}$ in which $z \equiv\left(n_{s}-\mu\right) / \beta$, where $\mu$ is the mode of the probability distribution, i.e., the highest probability point, and $\beta$ is the scale of the function. Furthermore, the median of the extremes of an exponentially distributed process follows $m=1 / \lambda\{\ln n-\ln [\ln (2)]\}$ in which $n$ is the number of samples [33].

In Fig. 4(e), for several system sizes we plot the distribution of all compositional $A$-particle fluctuations $P\left(n_{A}\right)$. The dashed line indicates that, for large $n_{A}$, $P\left(n_{A}\right)$ exhibits an exponential decay virtually independent of the system size, as expected. This motivates us to fit the Gumbel distribution to $P\left(n_{s}\right)$ in Fig. 4(a) (full lines). For $N \gtrsim 10000$, the agreement is remarkable. Moreover, the median of the fitted Gumbel distributions $\left\langle n_{s}\right\rangle$ exhibits a logarithmic dependence on the system size $N$ as shown in Fig. 4(d). We conclude that because the scaling and distribution follows the Gumbel distribution, the largest compositional fluctuation is consistent with exponentially distributed fluctuations.

In fact, from Fig. 4(e) we find that the decay constant of the exponential is $\lambda \approx 0.22$. This value of $\lambda$ corresponds to a completely random distribution of $A$ and $B$ particles, indicating that the large regions of one species are mainly entropic and thus present irrespective of the particular system; the probability to find $n A$ particles in a cluster of $n$ particles, assuming indistinguishable $A$ and $B$ particles, is $P(n)=\left(x_{A}\right)^{n}=\exp [n \ln (0.8)] \approx \exp (-0.22 n)$.

Merely demonstrating the existence and size of these fluctuations is, of course, not sufficient. We need to show also that they are sufficiently long-lived to initiate the crystallization as well. In order to address this question, we now consider dynamics. At $T=0.40$, as noted above, the structural relaxation time $\tau_{\alpha}=2.91 \times 10^{5}$. This is wildly in excess of the nucleation time in the one-component system at these temperatures, which is $\tau_{X} \approx 41$ for a system size of $N=13500$ [34]. Thus, since the lifetime of the compositional fluctuations must be on the order of $\tau_{\alpha}$ at least, and we do not see any signs of phase separation, i.e., other mechanisms of crystallization [see Fig. 4(c) and composition-composition correlation functions in the SM [32] ], we conclude that the compositional fluctuations we identify lead to crystallization.

Before we explore the compositional fluctuations for other systems, we provide some considerations as to the crystallization mechanism. One alternative possibility is enhancement of nucleation related to density fluctuations. Now the liquid-gas binodal has been measured as lying at a temperature not much less than $T \sim 0.40$ to which we simulate [35]. It is conceivable that some density fluctuations related to the proximity of liquid-gas phase separation might act to enhance nucleation, as is known for proteinlike systems $[18,19]$. However, the system is not in or near the two-phase region: the density of $\rho=1.204$ we consider is much higher than the critical isochore (around 0.3). In the 
SM [32] we investigate but see little evidence for density fluctuations [35]. In any case, any such nucleation enhancement would still need to invoke a mechanism for $A-B$ demixing, which is absent. Indeed, to observe demixing in similar binary systems, one needs to weaken the interaction between the species so that it is again nonadditive but weaker than the additive case, i.e., a positive enthalpy of mixing [36]. In fact, we see very little evidence for $A-B$ demixing (see SM [32]), (the compositional fluctuations we have discussed notwithstanding). In short, we provide evidence that the compositional fluctuations we identify here are unrelated to the density fluctuations known to enhance nucleation in (effective onecomponent) proteinlike systems $[18,19]$.

We also consider the consequences of our choice of an instantaneous quench protocol (see the Appendix). In Fig. S3 of the SM [32], we see that the median of the largest region of liquid $A$ particles $\left\langle n_{s}(T)\right\rangle$ shows very little dependence on temperature. Thus, as the system samples from a nearly temperature-independent distribution and due to the long mean nucleation time for $T>0.40$ (more than $100 \tau_{\alpha}$ ), we argue that our quenching protocol does not affect our conclusions to any significant extent. The independence with respect to temperature is intriguing: we interpret this in the context that the structure of the liquid is dominated by the hard core [37], in which case a weak temperature dependence is expected.

\section{DEPENDENCE OF FLUCTUATIONS ON SYSTEM COMPOSITION}

We now consider other compositions of the KA mixture. The temperature independence of the compositional fluctuations suggests that the scaling leading to large compositional fluctuations may be identified at high temperature where timescales are amenable to computer simulation, without recourse to simulations of the deeply supercooled liquid. This suggests that it may be possible to use our approach to predict the glass-forming ability of mixtures in the liquid state.

Usually, as above, the 4:1 KA mixture is simulated, but upon changing the composition to be more equimolar, we expect smaller regions of pure $A$ particles. We focus on the 2:1 KA mixture at zero pressure and at the higher temperature of $T=0.80$, where the relaxation times for $4: 1$ and 2:1 KA are comparable [38]. We also considered the $3: 1$ composition, which turns out to lie close to the $2: 1$ system. In Fig. 4(d), we see that at zero pressure and $T=0.80$ the $4: 1$ mixture has a value of $\left\langle n_{s}\right\rangle$ very similar to that at which we see crystallization $(T=0.40)$. We infer that the change in pressure also has little effect on the compositional fluctuations, which is reasonable as they are largely random, according to the exponential distribution [Fig. 4(e)].

As expected, the 2:1 KA mixture in Fig. 4(d) has very much smaller values of $\left\langle n_{s}\right\rangle$, as its composition is closer to equimolar. To predict where crystallization might occur, we fit each composition to a logarithmic increase as indicated by the dashed lines in Fig. 4(d). From this we find that the 2:1 KA system reaches the value of $\left\langle n_{s}(N)\right\rangle=31$ (corresponding to the $4: 1$ system with $N \approx 10000$ ) at a system size of $N=1.2 \times 10^{9}$. Thus we expect that, for the mechanism of crystallization we consider here, the 2:1 composition should be a very much better glass former than the usual 4:1 system. We confirm this by very lengthy simulations of the $2: 1$ (and 3:1) KA systems at comparable supercoolings (i.e., $T=0.40$ for $\mathrm{KA} 4: 1$ ) and $N=10000,100000$, and 1000000 , where no crystallization was observed. We simulated around $9 \times 10^{9}$ time steps for $N=10000$ and 100000 and $2 \times 10^{9}$ time steps of $N=1000000$.

Note that we are only considering the crystallization mechanism based on compositional fluctuations. While we expect the mechanism to be present in all mixtures, crystallization may be dominated by other, faster, mechanisms. For example, the 1:1 KA mixture forms a mixed bcc crystal quite rapidly [39].

\section{CRYSTALLIZATION IN COPPER ZIRCONIUM}

To address whether the mechanism described above pertains to other systems, we consider the metallic glass former copper zirconium. Here we use embedded atom model simulations (see the Appendix for more details). In Fig. 5(a), we show that, like the KA mixture, the extreme values of the composition fluctuations in $\mathrm{CuZr}$ also follow a Gumbel distribution. To determine the magnitude of the composition fluctuations in the liquid, we use the higher temperatures of $T=1500 \mathrm{~K}$ or $T=1270 \mathrm{~K}$, respectively, so that we can run the simulations quickly. Here we use the NPT ensemble with a Nose-Hoover thermostat [23]; see the Appendix for further details.

The system size dependence of $\left\langle n_{s}(N)\right\rangle$, where we consider fluctuations of the majority species, is shown in Fig. 4(d). Again we see the logarithmic scaling; moreover, compositions such as $\mathrm{Cu}_{64.5} \mathrm{Zr}_{35.5}$ exhibit weaker fluctuations compared to the KA model. Following our analysis of the 2:1 KA mixture, here we estimate that the system may be susceptible to crystallization at a system size of $N=3.5 \times 10^{16}$ (when $n_{s} \approx 31$ ). However, upon changing to the more asymmetric compositions $\mathrm{Cu}_{25} \mathrm{Zr}_{75}$ and $\mathrm{Cu}_{15} \mathrm{Zr}_{85}$, we see a marked increase in the fluctuations.

Given these larger fluctuations, the logarithmic scaling in Fig. 4(d) would indicate that the metallic glass former should crystallize for those more asymmetric compositions on simulation timescales already around $N=10000$, assuming similar behavior to KA. To investigate crystallization, we run simulations at lower temperatures for two compositions, $\mathrm{Cu}_{25} \mathrm{Zr}_{75}$ and $\mathrm{Cu}_{15} \mathrm{Zr}_{85}$. Here the system was first equilibrated at $T=2000$ or $1500 \mathrm{~K}$ depending on the composition and then rapidly cooled to the temperature of interest. For $\mathrm{Cu}_{25} \mathrm{Zr}_{75}$ and $\mathrm{Cu}_{15} \mathrm{Zr}_{85}$, and the temperatures at which we see crystallization, $T=900$ and $1100 \mathrm{~K}$, the 


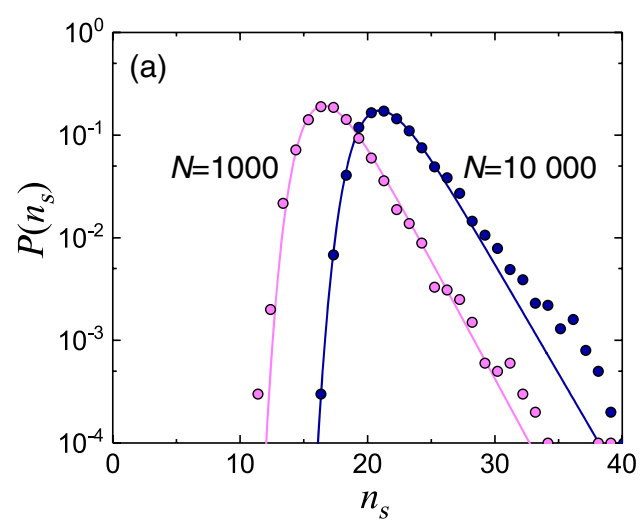

(b)

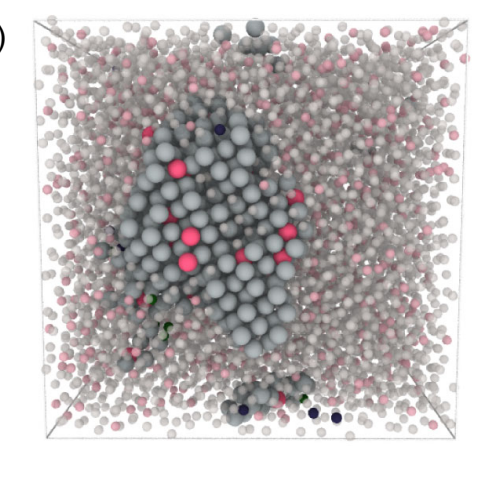

FIG. 5. Crystal nucleation in CuZr. (a) Compositional fluctuations of majority $\mathrm{Zr}$ atoms in $\mathrm{Cu}_{25} \mathrm{Zr}_{75}$ at $T=1500 \mathrm{~K}$. Lines are fits to a Gumbel distribution. (b) Nucleus in the early stage for $\mathrm{Cu}_{25} \mathrm{Zr}_{75}$ at $T=900 \mathrm{~K}$. Large gray particles are $\mathrm{Zr}$ (bcc), large pink are $\mathrm{Cu}$ (bcc), smaller gray and pink are liquid $\mathrm{Zr}$ and $\mathrm{Cu}$, respectively, and black are $\mathrm{Zr}$ in a local fcc environment as determined with bondorientational order parameter [25]. We see that the nucleus is dominated by $\mathrm{Zr}$.

cooling rates are $\Delta T / \Delta t=3.0 \times 10^{5}$ and $2.0 \times 10^{5} \mathrm{~K} / \mathrm{ps}$, respectively.

We find that $\mathrm{CuZr}$ indeed crystallizes with representative runs freezing after $930 \tau_{\alpha}$ and $95 \tau_{\alpha}$ for $\mathrm{Cu}_{25} \mathrm{Zr}_{75}$ and $\mathrm{Cu}_{15} \mathrm{Zr}_{85}$, respectively. Here we consider the bcc crystal, as the fcc and hcp are found only in trace quantities. In the snapshot in Fig. 5(b), we find that the nucleus for $\mathrm{Cu}_{25} \mathrm{Zr}_{75}$ is dominated by the majority species $\mathrm{Zr}$, in a manner similar to that in Fig. 1(a), although the growth is more rapid in the case of this binary metallic glass fomer (see SM [32]). Note that this higher rate of growth contrasts with slow growth previously observed in $\mathrm{CuZr}$ with respect to other metallic glass formers, for a binary crystal [40].

We thus infer that the mechanism for nucleation, at least for these compositions, is the same as that for crystallization in KA. Again, like KA, other mechanisms are also possible in which the crystal may be mixed [41,42]. However, we argue that we have presented a general crystallization mechanism in mixtures, which occurs in the absence of faster, specific, crystallization pathways.

\section{OUTLOOK}

Before concluding, we consider the consequences for the long-term stability of supercooled mixtures. We have shown that crystal nuclei are expected in mixtures in general. But by how much should they grow? By considering the KA mixture, the growth of fcc nuclei of $A$ particles will deplete the remaining liquid of $A$ particles. This depletion will tend to slow and may even arrest the growth of the one-component $A$ crystals. In the case of the KA system, we note that if the liquid approaches a $1: 1$ composition, then crystallization, not of the one-component fcc, but of the 1:1 composition bcc crystal, may be expected. We noted in the Introduction that the Ostwald rule of stages, generalized to mixtures, would provide pathways by which the nuclei may grow [20].
Given the small dimensions of the nuclei we find, and despite the developments we present here, our simulations are still small compared to experimental system sizes, and thus it seems reasonable to suppose that the final material may be composed of nanocrystals. Nanocrystals are known to have important consequences for the mechanical properties of glass-forming materials [43]. While this behavior has been seen in experiments [44], our work suggests that such nanocrystals may be rather prevalent in metallic glasses. Because identifying tiny crystalline regions is hard with $\mathrm{x}$-ray scattering, requiring techniques such as $3 \mathrm{D}$ atom probe tomography [44], nanobeam electron diffraction [45] or fluctuation TEM [46], it is possible that such nanocrystals may go undetected. The detection of such nanocrystals is an exciting avenue for future research.

\section{DISCUSSION AND CONCLUSIONS}

We have demonstrated a general mechanism of crystallization in multicomponent systems. Our large-scale simulations of the widely used Kob-Andersen model supercooled liquid reveal that it has a fatal flaw as a glass former which is general to mixtures. Local compositional fluctuations lead to regions populated only by one species. These regions can be larger than the critical crystal nucleus size of the one-component system under similar conditions. Nucleation in these regions is fast on the timescale of this deeply supercooled liquid, apparently requiring little rearrangement of the particles, as is known to be the case for hard spheres at deep supercooling [47,48]. Our findings are important, as the results we reveal here pose a fundamental challenge for the development of glass-forming materials: mixtures whose components crystallize easily are themselves inherently unstable to crystallization and thus ultimately compromised as glass formers. Our findings rationalize the empirical rule of thumb that increasing the number of components tends to increase glass-forming 
ablility, as the chances that a critical nucleus of one particular species is formed are reduced in that case.

We find a scaling with system size which, once parametrized, may be used to predict the largest system which is stable against crystallization, and therefore the largest pieces of amorphous material which can be prepared from a given mixture. That the compositional fluctuations are rather random and insensitive to temperature suggests that simulations in the liquid at higher temperature where the dynamics are much faster may be used to predict the system size at which crystallization may be expected. We have demonstrated this principle using the 2:1 (and 3:1) KA mixtures and have predicted that both can reach system sizes, for comparable simulation times and supercoolings, very much larger than the usual $4: 1$ mixture before crystallization occurs. These compositions may thus be used when a better glass former is needed in simulations than the standard $4: 1$ model.

The binary model we use demonstrates the use of a mixture to suppress crystallization, as is typically employed in metallic and inorganic glass formers and is encountered in vitreous magmas. Although prevalent and accessible to computer simulation for the model systems we consider, we expect the same mechanism will operate for more general binary mixtures, and indeed for multicomponent systems frequently employed in the quest for ever-better glassforming alloys [7]. We demonstrate this by considering the well-studied $\mathrm{CuZr}$ metallic glass former, which exhibits the same behavior. Experimental evidence in support of the mechanism we find has been seen in some metallic glasses [44] and we suggest that the presence of such nanocrystals as we identify here would be worth investigating further in metallic glasses.

Crystallization via compositional fluctuations thus forms a lower bound to nucleation: other mechanisms involving more complex crystal structures may prove faster, as indeed seems to be the case for some models [41,49] and for certain compositions of the Kob-Andersen [39] and $\mathrm{CuZr}$ [41] models considered here. Nevertheless, we have shown that liquids which rely on mixing for their stability against crystallization are fundamentally compromised and provide a principle by which their glass-forming ability may be optimized.

In addition to the number of components, crystallization may be suppressed in alloys by the use of systems with a negative heat of mixing. Here, the Kob-Andersen mixture is engineered in that way, precisely to inhibit crystallization. However, our analysis in Sec. IV suggests that such negative heat of mixing has little impact: the Gumbel distribution assumes that the two species are randomly distributed in space, so given its success in describing the statistics, we infer that our analysis is robust to the case where there is a negative heat of mixing. Noting that small size disparities will permit rapid crystallization, and that for certain size ratios binary crystals form an additional route to crystallization as noted in Sec. VII [14,42], we expect that increasing the size ratio will inhibit crystallization. We leave the prospect of a detailed analysis of the role of size disparity for the future.

In addition to the metallic glasses we consider here, an intriguing case is aqueous ionic solutions. Here, crystallization of water occurs through segregation to ion-rich and ion-poor regions, the latter being where the ice nucleates, which appears similar to what we observe here, for the $A$ particles [3]. However, the various anomalies in the thermodynamic behavior of water, not least increasing fluctuations, which may be related to an (avoided) liquid-liquid transition [50,51], mean that further study of that system would be needed to ascertain whether the mechanism we have identified here dominates water crystallization in some aqueous solutions.

\section{ACKNOWLEDGMENTS}

It is a pleasure to thank Nick Bailey, Daniele Coslovich, Daan Frenkel, Felix Höfling, Peter Harrowell, Takeshi Kawasaki, Rob Jack, Ken Kelton, Heidy Mader, Kunimasa Miyazaki, John Russo, Hajime Tanaka, and Jianguo Wang for stimulating discussions. C.P.R. acknowledges the Royal Society, and Kyoto University SPIRITS fund, and European Research Council (ERC consolidator grant NANOPRS, Project No. 617266) for financial support. T. S. I. acknowledges support from a JSPS Postdoctoral Fellowship under which part of this work was carried out. T. S. I., T. B.S., and J.C.D. are supported by the VILLUM Foundation's Matter Grant (No. 16515). The authors are grateful to Ioatzin Rios de Anda for inspirational help with the figures. LAMMPS simulations was carried out using the computational facilities of the Advanced Computing Research Centre, University of Bristol. RUMD simulations were out carried using the HPC cluster at Department of Science and Environment, Roskilde University.

\section{APPENDIX: METHODS}

\section{Simulation and model details}

We simulate the KA binary mixture in the $N V T$ ensemble (Nose-Hoover thermostat [23]) at $\rho=1.204$ using the Roskilde University molecular dynamics (RUMD) package [52] optimized for highly efficient GPU simulations; the longest simulations took more than 100 days. The interatomic interactions of the 4:1 binary mixture are defined by $v_{i j}(r)=\epsilon_{\alpha \beta}\left[\left(\sigma_{\alpha \beta} / r\right)^{12}-\left(\sigma_{\alpha \beta} / r\right)^{6}\right]$ with parameters $\sigma_{A B}=0.80, \sigma_{B B}=0.88$ and $\epsilon_{A B}=1.50$, $\epsilon_{B B}=0.50(\alpha, \beta=A, B)$. The pair potential is cut and shifted at $r_{c}=2.5 \sigma_{\alpha \beta}$. We employ a unit system in which $\sigma_{A A}=1, \epsilon_{A A}=1$, and $m_{A}=m_{B}=1$. We study system sizes $N=125,250,500,1000,2000,4000,5000,7000$, $10000,20000,30000,50000,80000,100000$, and 200000 at $T=0.40$. Several different temperatures, 
$T=0.395, \quad 0.40, \quad 0.415, \quad 0.43,0.45$, are studied at $N=10000$. The protocol for studying crystallization in the KA mixture is identical for all temperatures and system sizes studied. We equilibrate at $T=2.00$ and then perform an instantaneous quench to low temperatures, simulating between $9 \times 10^{9}$ and $36 \times 10^{9}$ time steps after the quench $(\Delta t=0.0025)$. The cooling rates are $\Delta T / \Delta t=642,640$, 634,628 , and 620 in reduced units. For each temperature and system size we perform 10 independent quenches. Additionally, $4: 1,3: 1$, and $2: 1 \mathrm{KA}$ mixtures were also simulated in the $N V T$ ensemble at a mean pressure $\langle P\rangle=0$ and $T=0.80$, with $N=1000,5000$, and 10000 , at which the relaxation times of the systems are similar.

Simulations of $\mathrm{Cu}_{x} \mathrm{Zr}_{1-x}$ mixtures were performed in the NPT ensemble using a Nose-Hoover thermostat and barostat with the LAMMPS package $[23,53]$ and compositions of $x=15 \%, 20 \%, 25 \%, 35.5 \%$, and $64.5 \%$. The Finnis-Sinclair embedded atom model method was applied [54], simulating at a pressure $P=0(\Delta t=0.002 \mathrm{ps})$. Three system sizes were simulated, $N=1000,5000$, and 10000 , at high temperatures for composition statistics, and nucleation was studied for $N=10000$.

\section{Relaxation time determination}

For the KA model, we determine the relaxation time of the liquid $\tau_{\alpha}$ from the self-part of the intermediate scattering function $F_{s}(q, t) \equiv\langle\exp [i q \Delta \mathbf{r}]\rangle$ of the $A$ particles using the criterion $F_{s A}\left(q, \tau_{\alpha}\right)=0.2$; the length of the wave vector is $q=7.25$. A system size of $N=1000$ is used for these simulations to suppress nucleation but has a minor effect on $\tau_{\alpha}$. In the case where we cannot measure $\tau_{\alpha}$ directly in simulations due to extremely long simulation timescales, we extrapolate using a Vogel-Fulcher-Tamman fit (see $\mathrm{SM}$ for more details [32]). For $\mathrm{Cu}_{x} \mathrm{Zr}_{1-x}$ we obtained the intermediate scattering function from the $\mathrm{Zr}$ atoms and used a wave vector with $q \approx 26 \mathrm{~nm}^{-1}$.

\section{Identifying local structure}

To detect the fcc and hcp crystals, and the bicapped square antiprism liquid locally favored structure, we use the topological cluster classification (TCC), employed previously to identify local structures in the KA mixture [24]. That is to say, we carry out a standard Voronoi decomposition and seek structures topologically identical to geometric motifs of particular interest.

For the bcc crystal, we employ a bond-orientational order (BOO) parameter analysis [25]. For each particle $i$ we define complex order parameters $q_{l m}^{i} \equiv$ $1 / n_{b} \sum_{j=1}^{n_{b}} Y_{l m}\left(\theta_{i j}, \phi_{i j}\right)$, where $Y_{l m}$ is the spherical harmonic function with degree $l$ and order $m, \theta$ and $\phi$ are the spherical coordinates for the vector $\mathbf{r}_{i j} \equiv \mathbf{r}_{j}-\mathbf{r}_{i}$, and $n_{b}$ is the number of neighbors defined from the 12 nearest neighbors. We use the complex order parameters to differentiate between solid and liquid particles using the criteria that for at least 7 nearest-neighbor bonds the scalar product $\mathbf{q}_{6}^{i} \cdot \mathbf{q}_{6}^{j} /\left|\mathbf{q}_{6}^{i}\right|\left|\mathbf{q}_{6}^{j}\right|$ should be greater than 0.70 to be classified as a solid particle. $\mathbf{q}_{6}^{i}$ is a $(2 l+1)$-dimensional complex vector. The identity of each solid particle is then determined [55] using the third-order invariant order parameters,

$$
W_{l}^{i} \equiv \sum_{m_{1}, m_{2}, m_{3}=0}^{l}\left(\begin{array}{ccc}
l & l & l \\
m_{1} & m_{2} & m_{3}
\end{array}\right) \frac{Q_{l m_{1}}^{i} Q_{l m_{2}}^{i} Q_{l m_{3}}^{i}}{\left|\mathbf{Q}_{l}^{i}\right|^{3}},
$$

where the term in the parentheses is the Wigner $3-j$ symbol and $Q_{l m}^{i} \equiv 1 /\left(n_{b}+1\right) \sum_{i=1}^{n_{b}+1} q_{l m}^{i}$ is the average bond-orientational order parameter [26]. bcc particles are identified as all solid particles having $W_{6}^{i}>0$. We checked that the TCC and BOO methods for the detection of fcc and hep gave similar results.

[1] A. L. Greer and K.F. Kelton, Nucleation in Condensed Matter: Applications in Materials and Biology, 1st ed. (Pergamon, New York, 2010), Vol. 15.

[2] K. Cashman and J. Blundy, Degassing and Crystallization of Ascending Andesite and Dacite, Phil. Trans. R. Soc. A 358, 1487 (2000).

[3] G. Bullock and V. Molinero, Low-Density Liquid Water Is the Mother of Ice: On the Relation between Mesostructure, Thermodynamics and Ice Crystallization in Solutions, Faraday Discuss. 167, 371 (2013).

[4] L. Berthier and G Biroli, Theoretical Perspective on the Glass Transition and Amorphous Materials, Rev. Mod. Phys. 83, 587 (2011).

[5] A. Inoue and A. Takeuchi, Recent Development and Application Products of Bulk Glassy Alloys, Acta Mater. 59, 2243 (2011).

[6] N. Togashi, M. Ishida, N. Nishiyama, and A. Inoue, Wear Resistance of Metallic Glass Bearings, Rev. Adv. Mater. Sci. 18, 93 (2008).

[7] Y.Q. Cheng and E. Ma, Atomic-Level Structure and Structure-Property Relationship in Metallic Glasses, Prog. Mater. Sci. 56, 379 (2011).

[8] J. D. Honeycutt and H. Andersen, Small System Size Artifacts in the Molecular Dynamics Simulation of Homogeneous Crystal Nucleation in Supercooled Atomic Liquids, J. Phys. Chem. 90, 1585 (1986).

[9] F. H. Stillinger, A Topographic View of Supercooled Liquids and Glass Formation, Science 267, 1935 (1995).

[10] J. D. Stevenson and P. Wolynes, The Ultimate Fate of Supercooled Liquids, J. Phys. Chem. A 115, 3713 (2011).

[11] W. Kauzmann, The Nature of the Glassy State and the Behavior of Liquids at Low Temperatures, Chem. Rev. 43, 219 (1948).

[12] T. Hecksher, A. I. Nielsen, N. B. Olsen, and J. C. Dyre, Little Evidence for Dynamic Divergences in Ultraviscous Molecular Liquids, Nat. Phys. 4, 737 (2008).

[13] H. Tanaka, Possible Resolution of the Kauzmann Paradox in Supercooled Liquids, Phys. Rev. E 68, 011505 (2003).

[14] K. Zhang et al., J. Schroers, M. D. Shattuck, and C. S. O'Hern, Computational Studies of the Glass-Forming Ability 
of Model Bulk Metallic Glasses, J. Chem. Phys. 139, 124503 (2013).

[15] S. Toxvaerd, U. R. Pedersen, T. B. Schroder, and J. C. Dyre, Stability of Supercooled Binary Liquid Mixtures, J. Chem. Phys. 130, 224501 (2009).

[16] A. Ninarello, L. Berthier, and D. Coslovich, Models and Algorithms for the Next Generation of Glass Transition Studies, Phys. Rev. X 7, 021039 (2017).

[17] D. Coslovich, M. Osawa, and L. Berthier, Local Order and Crystallization of Dense Polydisperse Hard Spheres, J. Phys. Condens. Matter 30, 144004 (2018).

[18] P. R. Ten Wolde and D. Frenkel, Enhancement of Protein Crystal Nucleation by Critical Density Fluctuations, Science 277, 1975 (1997).

[19] P. G. Vekilov, The Two-Step Mechanism of Nucleation of Crystals in Solution, Nanoscale 2, 2346 (2010).

[20] J. W. P. Schmelzer, V. M. Fokin, A. S. Abyzov, E. D. Zanotto, and I. Gutzow, How Do Crystals grow in GlassForming Liquids? Ostwld's Rule of Stages and Beyond, Int. J. Appl. Glass Sci. 1, 16 (2010).

[21] W. Kob and H. C. Andersen, Scaling Behavior in the BetaRelaxation Regime of a Supercooled Lennard-Jones Mixture, Phys. Rev. Lett. 73, 1376 (1994).

[22] P. S. Salmon and A. Zeidler, Identifying and Characterising the Different Structural Length Scales in Liquids and Glasses: An Experimental Approach, Phys. Chem. Chem. Phys. 15, 15286 (2013).

[23] M. P. Allen and D. J. Tildesley, Computer Simulation of Liquids, 2nd ed. (Oxford University Press, Oxford, 2017).

[24] A. Malins, J. Eggers, H. Tanaka, and C. P. Royall, Lifetimes and Lengthscales of Structural Motifs in a Model Glassformer, Faraday Discuss. 167, 405 (2014).

[25] P. J. Steinhardt, D. R. Nelson, and M. Ronchetti, BondOrientational Order in Liquids and Glasses, Phys. Rev. B 28, 784 (1983).

[26] W. Lechner and C. Dellago, Accurate Determination of Crystal Structures Based on Averaged Local Bond Order Parameters, J. Chem. Phys. 129, 114707 (2008).

[27] J. Taffs, S. R. Williams, H. Tanaka, and C. P. Royall, Structure and Kinetics in the Freezing of Nearly Hard Spheres, Soft Matter 9, 297 (2013).

[28] U. K. Nandi, A. Banerjee, B. Chakrabarty, and S. M. Bhattacharyya, Composition Dependence of the Glass Forming Ability in Binary Mixtures: The Role of Demixing Entropy, J. Chem. Phys. 145, 034503 (2016).

[29] U. R. Pedersen, T. B. Schröder, and J. C. Dyre, Phase Diagram of Kob-Andersen-Type Binary Lennard-Jones Mixtures, Phys. Rev. Lett. 120, 165501 (2018).

[30] L. Filion, R. Ni, D. Frenkel, and M. Dijkstra, Simulation of Nucleation in Almost Hard-Sphere Colloids: The Discrepancy between Experiment and Simulation Persists, J. Chem. Phys. 134, 134901 (2011).

[31] R. Strey, J. Wedekinda, and D. Reguera, New Method to Analyze Simulations of Activated Processes, J. Chem. Phys. 126, 134103 (2007).

[32] See Supplemental Material at http://link.aps.org/ supplemental/10.1103/PhysRevX.9.031016 for Supplemental figures and analysis of density-density and compositioncomposition fluctuations via the respective structure factor.
[33] A. Bovier, Extreme Values of Random Processes, http:// citeseerx.ist.psu.edu/viewdoc/download?doi=10.1.1.703 $.3866 \&=$ rep $1 \&=$ pdf.

[34] L. Báez and P. Clancy, The Kinetics of Crystal Growth and Dissolution from the Melt in Lennard-Jones Systems, J. Chem. Phys. 102, 8138 (1995).

[35] V. Testard, L. Bethier, and W. Kob, Intermittent Dynamics and Logarithmic Domain Growth During the Spinodal Decomposition of a Glass-Forming Liquid, J. Chem. Phys. 140, 164502 (2014).

[36] S. Roy, S. Dietrich, and F. Höfling, Structure and Dynamics of Binary Liquid Mixtures Near Their Continuous Demixing Transitions, J. Chem. Phys. 145, 134505 (2016).

[37] J. D. Weeks, D. Chandler, and H. C. Andersen, Role of Repulsive Forces in Determining the Equilibrium Structure of Simple Liquids, J. Chem. Phys. 54, 5237 (1971).

[38] P. Crowther, F. Turci, and C. P. Royall, The Nature of Geometric Frustration in the Kob-Andersen Mixture, J. Chem. Phys. 143, 044503 (2015).

[39] J. Fernández and P. Harrowell, Crystal Phases of a GlassForming Lennard-Jones Mixture, Phys. Rev. E 67, 011403 (2003).

[40] C. Tang and P. Harrowell, Anomalously Slow Crystal Growth of the Glass-Forming Alloy CuZr, Nat. Mater. 12, 507 (2013).

[41] R. E. Ryltsev, B. A. Klumov, N. M. Chtchelkatchev, and K. Y. Shunyaev, Cooling Rate Dependence of Simulated $\mathrm{Cu}_{64.5} \mathrm{Zr}_{35.5}$ Metallic Glass Structure, J. Chem. Phys. 145, 034506 (2016).

[42] R. E. Ryltsev, B. A. Klumov, N. M. Chtchelkatchev, and K. Y. Shunyaev, Nucleation Instability in Super-Cooled Cu-Zr-Al Glass-Forming Liquids, arXiV:1809.00198.

[43] A. Inoue, W. Zhang, T. Tsurui, A. R. Yavari, and A. L. Greer, Unusual Room-Temperature Compressive Plasticity in Nanocrystal-Toughened Bulk Copper-Zirconium Glass, Philos. Mag. Lett. 85, 221 (2005).

[44] K. K. Sahu, N. A. Mauro, L. Longstreth-Spoor, D. Saha, Z. Nussinov, M. K. Miller, and K. F. Kelton, Phase Sparation Mediated Devitrification of $\mathrm{Al}_{88} \mathrm{Y}_{7} \mathrm{Fe}_{5}$ Glasses, Acta Mater. 58, 4199 (2010).

[45] A. Hirata, L. J. Kang, T. Fujita, B. Klumov, K. Matsue, M. Kotani, A. R. Yavari, and M. W. Chen, Geometric Frustration of Icosahedron in Metallic Glasses, Science 341, 376 (2013).

[46] B.-S. Lee, G. W. Burr, R. M. Shelby, S. Raoux, C. T. Rettner, S. N. Bogle, K. Darmawikarta, S. G. Bishop, and J. R. Abelson, Observation of the Role of Subcritical Nuclei in Crystallization of a Glassy Solid, Science 326, 980 (2009).

[47] E. Sanz, C. Valeriani, E. Zaccarelli, W. C. K. Poon, M. E. Cates, and P. N. Pusey, Avalanches Mediate Crystallization in a Hard-Sphere Glass, Proc. Natl. Acad. Sci. U.S.A. 111, 75 (2014).

[48] T. Yanagishima, J. Russo, and H. Tanaka, Common Mechanism of Thermodynamic and Mechanical Origin for Ageing and Crystallization of Glasses, Nat. Commun. 8, 15954 (2017).

[49] U. R. Pedersen, T. B. Schroder, J. C. Dyre, and P. Harrowell, Geometry of Slow Structural Fluctuations in a Supercooled Binary Alloy, Phys. Rev. Lett. 104, 105701 (2010).

[50] E. B. Moore and V. Molinero, Growing Correlation Length in Supercooled Water, J. Chem. Phys. 130, 244505 (2009). 
[51] J. C. Palmer, F. Martelli, Y. Liu, R. Car, A. Z. Panagiotopoulos, and P. G. Debenedetti, Metastable Liquid-Liquid Transition in a Molecular Model of Water, Nature (London) 510, 385 (2014).

[52] N. P. Bailey et al., RUMD: A General Purpose Molecular Dynamics Package Optimized to Utilize GPU Hardware Down to a Few Thousand Particles, SciPost Phys. 3, 038 (2017).
[53] S. Plimpton, Fast Parallel Algorithms for Short-Range Molecular Dynamics, J. Comput. Phys. 117, 1 (1995).

[54] M. I. Mendelev, M. J. Kramer, R. T. Ott, D. J. Sordelet, D. Yagodin, and P. Popel, Development of Suitable Interatomic Potentials for Simulation of Liquid and Amorphous $\mathrm{Cu}-\mathrm{Zr}$ Alloys, Philos. Mag. 89, 967 (2009).

[55] J. Russo and H. Tanaka, The Microscopic Pathway to Crystallization in Supercooled Liquids, Sci. Rep. 2, 505 (2012). 